\title{
Ring rolling with variable wall thickness
}

Christopher J Cleaver ${ }^{1}$, Matthew R Arthington ${ }^{2}$, Sharareh Mortazavi ${ }^{1}$, Julian M Allwood ${ }^{1}(1)$.

1. Department of Engineering, University of Cambridge, Cambridge, UK,

2. Department of Engineering Science, University of Oxford, Oxford, UK

Ring rolling processes today produce axisymmetric rings, wasting material, energy and labour if non-axisymmetric components such as eccentric bearing races and bossed pipe fittings are required. A new process is proposed to roll rings with variable wall thickness. In this work, roll gaps and speeds are controlled online in physical experiments to achieve a defined variable wall thickness, enabled by photogrammetry to capture the ring's shape and position. The trials revealed two new process limits for which new analytical explanations have been developed: a maximum rate of change of thickness around the circumference and a loss of circularity.

Rolling, Process-Control, Ring Rolling.

\section{Introduction}

Until now Ring Rolling has been used to produce notionally axisymmetric rings. Yet, if non-axisymmetric features are required in a component they must be machined from larger rings, wasting material, energy and labour inputs and increasing downstream machining burden. Such components could include eccentric bearing races or rings with one or more bosses around the circumference; both broadly circular but with variable wall thickness - as suggested in Figure 1. If it was possible to control wall thickness and/or curvature around the ring during ring rolling, this could be valuable to customers of such components.
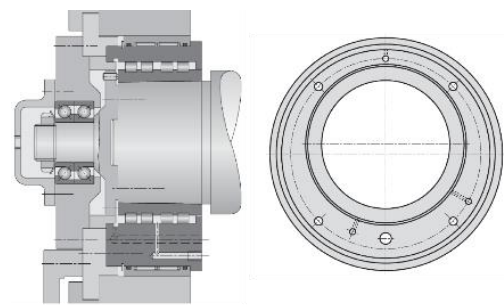

Triple Ring Eccentric Bearing, FAG Industrial Bearings AG[1]

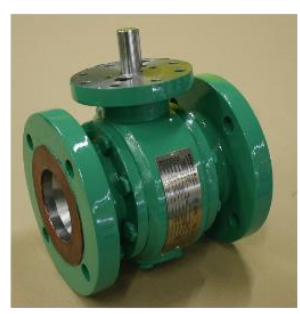

Side Entry Trunion Ball Valve, Erreesse S.r.l. [2]
Figure 1. Possible uses of variable thickness ring rolling

In the typical ring rolling process illustrated in Figure 2 a pierced preform is deformed between two pairs of rolls acting on the radial and axial surfaces. The radial roll pair comprise a powered forming roll, and idle mandrel and the axial roll pair are an upper and lower axial roll.

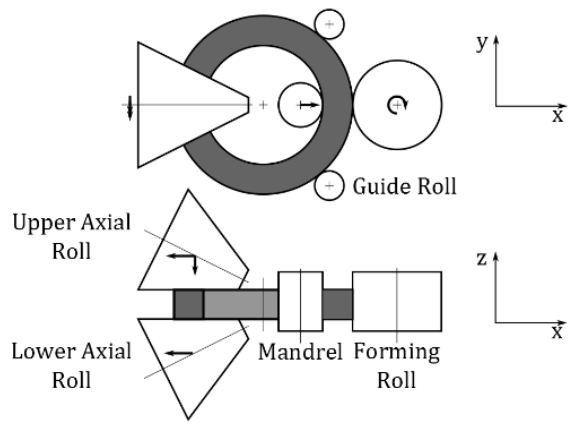

Figure 2. Typical radial-axial ring rolling process
A review of research into ring rolling in German and English languages $[3,4]$ showed that effort has historically centred on understanding and controlling the conventional process [e.g. 5,6]; recent innovations include incremental techniques for producing profiled rings [e.g. 7,8]. However, despite related applications in fields such as plate rolling [9], variable thickness ring rolling has not yet appeared in the literature. This paper describes how rolled rings could be produced with variable wall thickness for the first time.

\section{Method for creating variable wall thickness rings}

A novel method for production of rings with variable wall thickness is proposed and implemented on a model machine.

\subsection{Experimental set-up}

Experiments are performed on a desktop-scale ring rolling machine at the University of Cambridge, as shown in Figure 3 [10]. The machine uses modelling clay as a model material. Similar materials have been used previously for predicting geometrical outcomes in hot metal working processes [11].

The principal set-up and degrees of freedom are as described in Figure 2. Two differences are that guide rolls are not used and the ring is instead centred along the y-axis by differential speed control of the axial roll (discussed below). To simplify sensing, the radial roll pair is moved so the ring centre remains stationary along the $\mathrm{x}$-axis, but this does not affect the process mechanics.

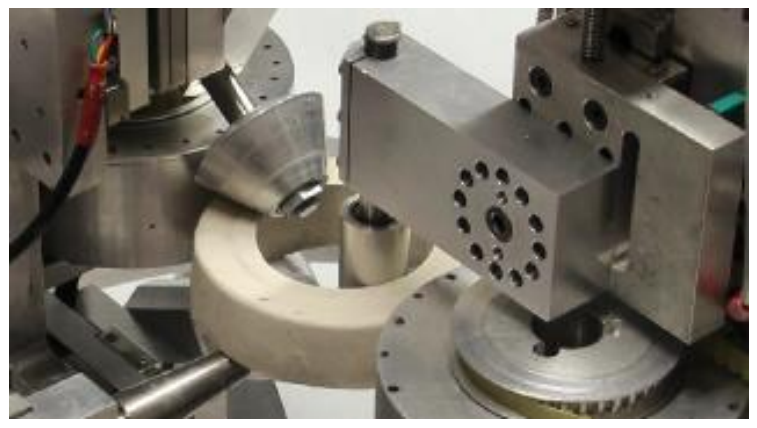

Figure 3. Model ring rolling machine at University of Cambridge

The model material is a proprietary oil-clay mixture produced by Newclay Products Ltd, UK. To make the preform, the material is heated in a water bath, kneaded and pressed in a closed mould. 
The material behaviour shown in Figure 4 is similar to hightemperature steel [11]; elastic deformation is followed by strainrate dependent plasticity with little strain hardening. The strain rate in the experiment, around $0.07 / \mathrm{s}$, is similar to that for the lowest curve in figure 4.

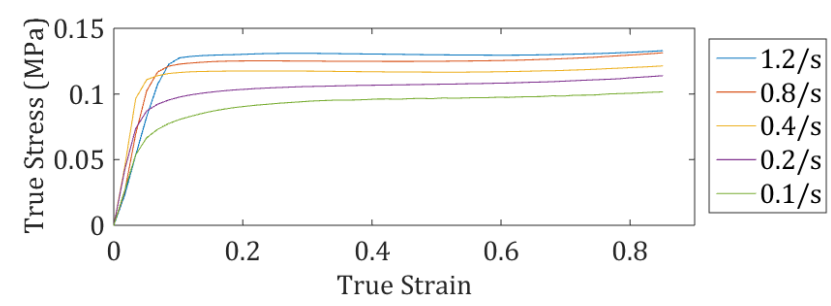

Figure 4. Modelling clay compression tests on cylindrical specimens

\subsection{Measuring the current state of ring geometry}

To control the ring's radial wall thickness, its current geometric state must be measured to provide feedback to the controller: an open loop approach to control would probably fail since the rotation of the ring is difficult to predict with sufficient accuracy.

Measurement is achieved by a calibrated optical camera mounted above the ring, as described in [12]. It measures thickness around the circumference by standard edge detection techniques and infers the location of the current ring centre and midline in the camera's frame of reference. The nominal midline radius is estimated around the circumference as the distance from ring centre to the midline.

In contrast to conventional ring rolling, the ring rotation must be monitored as well as its thickness, to allow precise control of thickness and strain around the ring circumference. To achieve this, 12 coloured markers are placed on the top ring surface and their location detected by changes in hue around the ring midline.

\subsection{Control of the process}

The control of variable wall thickness requires a different approach to that used previously. Conventionally, the radial roll gap is smoothly controlled to achieve a target rate of ring diameter growth. In variable wall thickness rolling, the roll gap must change dynamically to accommodate differences in thickness around the circumference whilst also reducing the overall mean ring thickness as the diameter grows.

An outline of the approach is shown in Figure 5. The target shape and initial preform are defined first. The target shape (which would in principle include a machining allowance) is specified as a final thickness distribution around the circumference, and the ring has a constant, specified, midline radius. The process controller then calculates a target thickness for each current material point around the circumference, assuming that volume is conserved during deformation, that plane rectangular sections remain plane and rectangular and that there is no axial flow. If the material behaves as expected then the final radius and thickness distribution will be as intended.

A schedule of planned reduction is required. Early trials highlighted three main problems. The first was complete slipping of the forming roll. In Section 4.1 it is shown that this can be avoided if rates of thickness change in the target ring are constrained and the reduction per pass is limited. Secondly, if the reduction is too small then the rolls lose contact with the ring this is avoided by imposing a minimum reduction, set to achieve the yield strain through the thickness. Finally, circularity can be compromised if the forming of the thickest sections completes ahead of the rest of the ring; therefore the strain was scheduled so that the deformation at all points around the circumference is completed after the same number of passes.

To cope with disturbances in incoming ring thickness and avoid violating process limits the controller is designed to track a schedule of through-thickness strain rather than exit thickness. The actual duration of processing is therefore set by the controller and may exceed the targeted number of revolutions.

In early trials without axial or guide rolls the variable thickness rings lost circularity regardless of reduction scheduling. This is unlike conventional rolling and is analysed Section 4.2 where it is shown that use and control of axial rolls can improve circularity. The forming roll speed and axial roll gap are both set to be constant throughout the process.

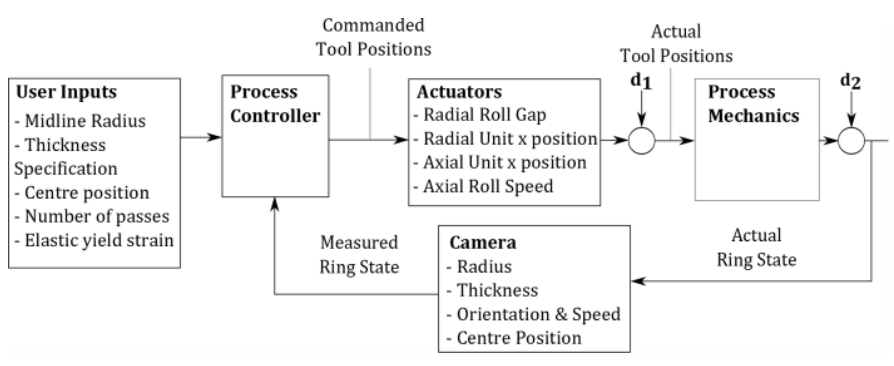

Figure 5. Block diagram of variable thickness controller

\section{Demonstration of the new method in two test cases}

More than 50 trials have been conducted with the process; of which two test cases are described to demonstrate the method.

\subsection{Demonstration part specifications}

The demonstration parts are shown in Figure 6; the first part is representative of the middle ring of a triple ring eccentric bearing, requiring a gradual change in thickness around an approximately-circular ring midline. Thickness strain varies from -0.14 to -0.52 . The second product is a ring with a single radial boss of boss arc angle, $\beta=5^{\circ}$, that is $58 \%$ thicker than the main circular ring. There are two transition regions over arc angle $\alpha=20^{\circ}$. The thickness strain varies from -0.05 to -0.51 .

Eccentric
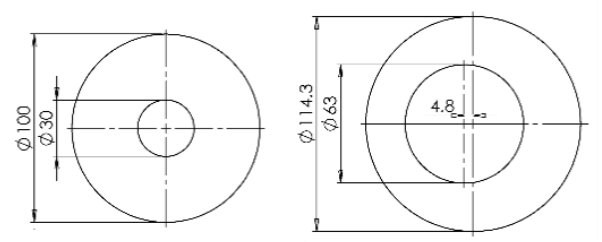

Boss
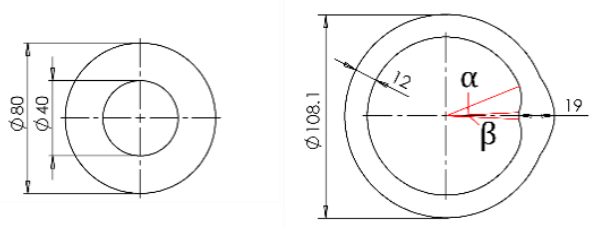

Figure 6: Preform (L) and target shape specifications (R)

\subsection{Results of controlled process}

The final ring shapes are shown in Figure 7. The main features of both parts clearly resemble the design in figure 6 .

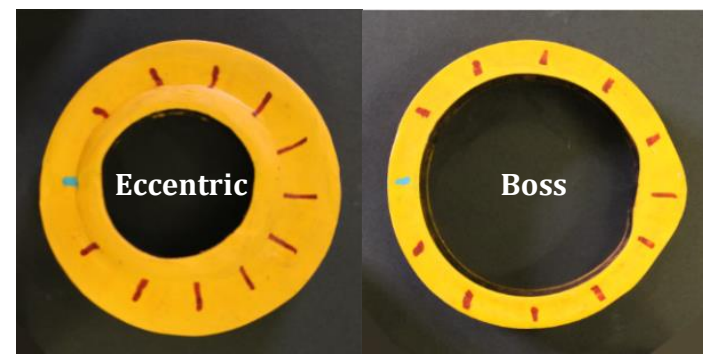

Figure 7. Demonstration parts produced by model ring rolling 
The error in thickness compared to the target specification is plotted in Figure 8, with the angle measured anticlockwise from the blue origin marker. Thickness errors are within $+2.5 /-1.0 \mathrm{~mm}$ for the eccentric part and +/- $1 \mathrm{~mm}$ for the boss part. The correct average radius has been reached in both cases. The larger error in the eccentric part can be accounted for by non-zero axial flow in the radial roll gap which is then reversed in the axial gap.

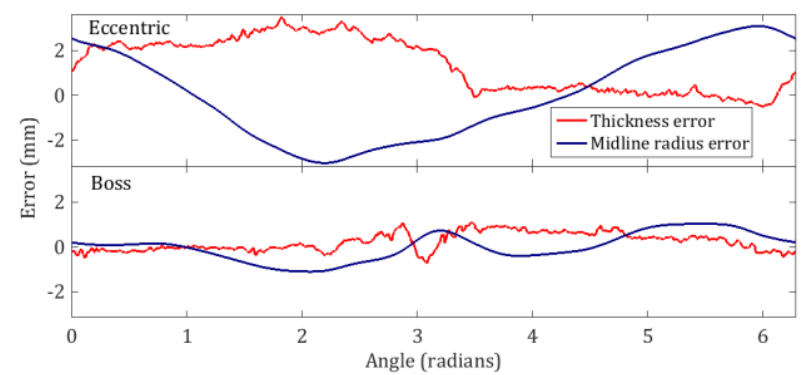

Figure 8. Error in thickness and radius for demonstration parts

The control system of Figure 5 acts to adjust the roll gap to match a scheduled strain path and its effect can be seen in Figure 9 where the commanded radial roll gap is compared against an open loop prediction that assumes the ring exits the gap with the same thickness as in the target schedule. For the eccentric gear ring, the open loop and commanded roll gaps are in phase, but due to machine compliance the actual gap is larger than expected during early rotations so the process duration is extended beyond the four revolutions targeted, and the radius continues to grow until the seventh revolution. The bossed ring schedule anticipates a constant gap for most of the revolution, reduced briefly as required to create the boss. Here, the commanded gap closely matches the scheduled six pass reduction as a result of the smaller roll force and hence less machine deflection.

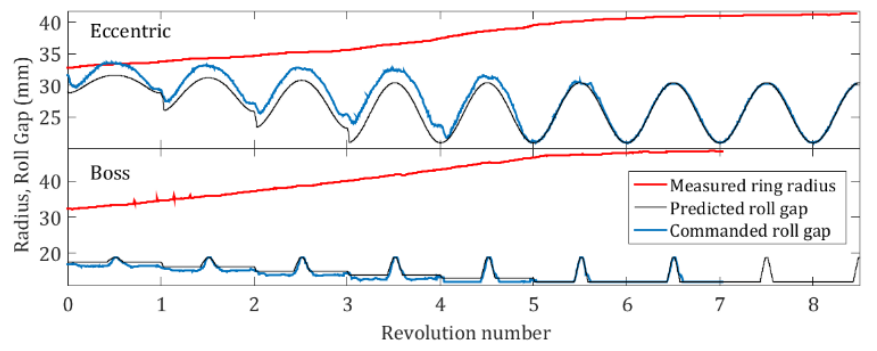

Figure 9. Commanded vs expected roll gap, radius and velocity

\section{Analysis of mechanical limitations}

The set of trials discussed in Section 3 revealed two novel features of the process mechanics: a limitation to the achievable rate of change of thickness around the circumference and noncircularity in the rings produced. These are now examined.

\section{Rate of thickness change}

The possibility of slipping between the forming roll and ring in the new process constrains the rate of thickness change around the circumference. In conventional ring rolling the ring is drawn through the roll bite provided the nominal draft, $\Delta \mathrm{h}$ (difference between incoming ring thickness and the roll gap) does not exceed a critical value, $\Delta \mathrm{h}_{\max }[12]$. However in variable thickness rolling the contact geometry can differ significantly and can lead to slip even if this condition is met. For instance, in early trials a step change was targeted but could not be achieved due to slipping as shown in Figure 10.

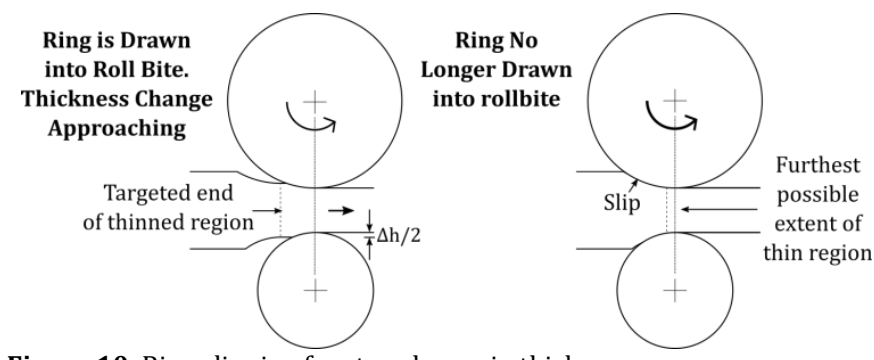

Figure 10. Ring slipping for step change in thickness

Instead, a smooth transition in thickness must be specified. For example a linear change in thickness giving constant slope angle, $\psi$ is shown in Figure 11. In this case, slipping is avoided provided $\psi$ is less than a critical value $\psi_{\text {crit }}$ at which point the y-component of traction, $\mathrm{T}_{1}$, can no longer exceed the $\mathrm{y}$-component of pressure $\left(\mathrm{P}_{1} \& \mathrm{P}_{2}\right)$ and the roll slips. An analytical approach similar to that described by Lin et al. [13] was used to determine $\psi_{\text {crit. }}$

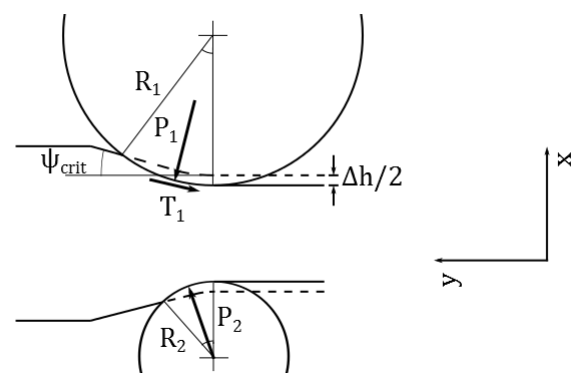

Figure 11. Roll bite force diagram for linear change in thickness

An assumed force and roll bite geometry is set up as in Figure 11. Force equilibrium along the $\mathrm{x}$-axis and $\mathrm{y}$-axis and either a Coulomb friction and or Tresca sticking-friction models was used. For the former, the critical slope, $\psi_{\text {crit, }}$ is found by solving equation 1 :

$$
\begin{aligned}
2 \tan ^{-1}(\mu)=2 \psi & +\cos ^{-1}\left[\left(1-\frac{\Delta h}{2 R_{1}}\right) \cos (\psi)\right] \\
& +\cos ^{-1}\left[\left(1-\frac{\Delta h}{2 R_{2}}\right) \cos (\psi)\right]
\end{aligned}
$$

This is evaluated in Table 1 for the rolling geometry in the boss ring demonstration, with the result used to report a maximum rate of thickness change $\tan \left(\psi_{\text {crit }}\right)$. The value of $\psi_{\text {crit }}$ depends on the draft, $\Delta \mathrm{h}$, which reduces to zero as $\Delta \mathrm{h}$ approaches $\Delta \mathrm{h}_{\max }$.

Table 1: Predicted maximum rate of thickness change $(\mathrm{mm} / \mathrm{mm})$

\begin{tabular}{cccc}
\hline \multirow{2}{*}{$\begin{array}{c}\text { Draft } \\
(\% \text { of }\end{array}$} & \multicolumn{3}{c}{ Max. rate of thickness change $(\mathrm{mm} / \mathrm{mm})$} \\
\cline { 2 - 4 }$\left.\Delta \mathrm{h}_{\max }\right)$ & Coulomb & Coulomb & Tresca \\
& $\mu=0.2$ & $2.72 \mathrm{~mm}$ & $4.59 \mathrm{~mm}$ \\
\hline$\Delta \mathrm{h}_{\max }$ & $0.74 \mathrm{~mm}$ & 0.39 & 0.67 \\
$0 \%$ & 0.20 & 0.28 & 0.53 \\
$25 \%$ & 0.15 & 0.19 & 0.38 \\
$50 \%$ & 0.10 & 0.10 & 0.21 \\
$75 \%$ & 0.05 &
\end{tabular}

$\mathrm{T}=12.0 \mathrm{~mm}, \mathrm{RF}=50.0 \mathrm{~mm}, \mathrm{RM}=10.0 \mathrm{~mm}$

Table 2: Targeted vs achieved rate of thickness change $(\mathrm{mm} / \mathrm{mm})$

\begin{tabular}{ccc}
\hline Targeted & Achieved - boss entry & Achieved - boss exit \\
\hline 0.42 & 0.35 & 0.46 \\
0.83 & 0.61 & 0.36 \\
1.67 & 0.49 & 0.54 \\
\hline
\end{tabular}

A set of three tests confirmed the maximum rate of change of thickness that could be achieved in practice. The results in Table 2 show a maximum rate of change of thickness of $0.61 \mathrm{~mm} / \mathrm{mm}$ when targeting $0.83 \mathrm{~mm} / \mathrm{mm}$. This agrees with the limit predicted in Table 1 with Tresca friction, the likely friction 
regime. In practice, the formation of scale may affect this result significantly.

\subsection{Loss of ring circularity}

A second novel feature of the process mechanics of variable thickness rolling is a loss of ring circularity. To produce a circular ring a uniform curvature change is required as the ring expands, yet in contrast to uniform thickness rolling, this can not be achieved without additional control.

To analyse the effect, a series of six shapes is produced from the same preform, without extra control. The shapes are similar to the bossed ring part in Figure 6, but with varying boss angle $\{\beta=$ $\left.0^{\circ}, 5^{\circ}, 15^{\circ}, 30^{\circ}, 60^{\circ}, 120^{\circ}\right\}$. The target thicknesses are adjusted so that each part has an equal midline radius, and the ratio of engineering strain in the boss to that in the main ring (8:1) is kept constant. The distance from midline to centre is estimated around the circumference according to Section 2.2. The ratio of the range of this distance to its average was used as a metric of circularity.

The resulting rings are shown in Figure 12a. Only the uniform thickness ring $\left(\beta=0^{\circ}\right)$ is close to circular; all rings with variable thickness have poor circularity, with errors of about $10 \%$ or greater as shown in Figure 13. Preliminary analysis suggests that curvature changes in ring rolling are governed by elastic moments at the entry and exit to the radial roll bite. In uniformthickness rolling a self-regulating moment is provided by the state of bending in the ring outside of the roll bite. However, this is no longer sufficient in the variable thickness process because the required moment varies around the ring. a. Uncontrolled

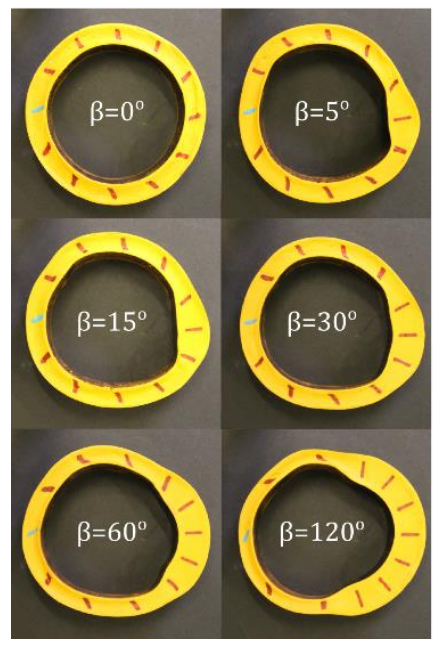

b. Controlled

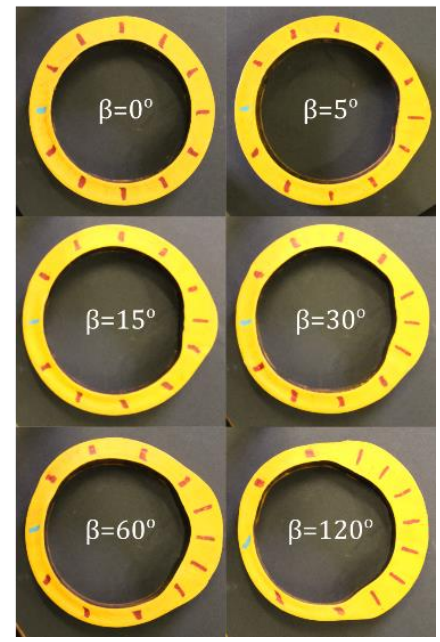

Figure 12. Final rings for a range of boss angles

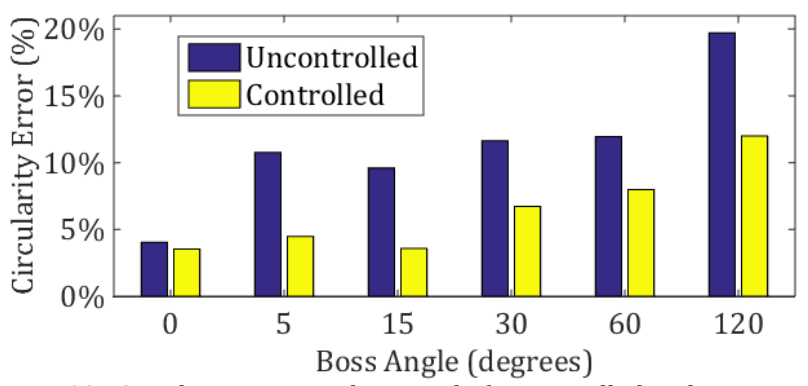

Figure 13. Circularity error vs boss angle for controlled and uncontrolled variable thickness ring rolling

Instead, a corrective moment is required. This could potentially be supplied by the action of guide rolls, axial rolls or both. The pronounced changes in thickness make it difficult to determine the correct positioning of guide rolls, and the simplest approach was to apply differential speed control to the axial rolls. Trials have shown that if tools act to centre the ring along the $y$-axis in Figure 2, the correct moment will be applied.

This approach was implemented with a Proportional controller to a second set of rings, as shown in Figure 12b. The results show that with centring, it has been possible to keep the radius error below $5 \%$, for narrower bosses with $\beta<30$ degrees. Whilst this is still high for practical use, it is similar to the uniform thickness ring and there is scope to improve this in future industrial trials.

\section{Discussion}

This paper has introduced a viable method for producing variable thickness rings which has been demonstrated with a model material with low force, low rolling speed and high friction. The paper has established two important limits that are likely to translate to an industrial setting.

The effect of variable thickness rolling on metallurgical properties of the ring is yet to be established. This may be important: for example, the ratio of maximum to minimum strains (up to 8:1) used here may not be possible in all materials. The sensing method may need further development for application to hot ring rolling.

More advanced curvature control could be developed, for example to shift the ring's nominal centre to other locations relative to the machine, enabling production of deliberately noncircular rings. This will be investigated in future research.

\section{Acknowledgements}

The first author is supported by an EPSRC I-Case Studentship (12220703), with Primetals Technologies Ltd. Dr Arthington and Prof. Allwood were supported by EPSRC grant EP/K018108/1.

\section{References}

[1] FAG Industrial Bearings AG (2003) FAG Triple Ring Eccentric Bearings in a Sheetfed Offset Press. Publ. No. WL 23502 EA

[2] Erreesse (2014). Workshop - Erreesse. N.p.,. Web. 19.01.2016.

[3] Allwood, J. M., Tekkaya, A. E., \& Stanistreet, T. F. (2005). The development of ring rolling technology - Part 1. Steel Research International, 76(2/3), 111-120.

[4] Allwood, J. M., Tekkaya, A. E., \& Stanistreet, T. F. (2005). The Development of Ring Rolling Technology - Part 2. Steel Research International, 76(7), 489-505.

[5] Hawkyard, J., \& Johnson, W. (1973). Analyses for roll force and torque in ring rolling, with some supporting experiments. Int. J. mech. Sci., 15, 873-893

[6] Kopp, R., Koppers, U., \& Wiegels, H. (1984). New Control System for Ring Rolling. Advanced Technology of Plasticity, 2, 803-807.

[7] Allwood, J., Kopp, R., \& Michels, D. (2005). The technical and commercial potential of an incremental ring rolling process. CIRP Annals, 2(1), 1-4.

[8] Tiedemann, I., Hirt, G., Kopp, R., Michl, D., \& Khanjari, N. (2007). Material flow determination for radial flexible profile ring rolling. Production Engineering, 1(3), 227-232

[9] Kopp, R. Wiedner, C. Meyer, A. (2005) Flexible Rolled Sheet Metal and Its Use in Sheet Metal Forming. Advanced Material Research, 6-8, pp. 81-92

[10] Stanistreet, T. F., Allwood, J. M., \& Willoughby, a. M. (2006). The design of a flexible model ring rolling machine. Journal of Materials Processing Technology, 177(1-3), 630-633

[11] Wanheim, T., Schreiber, M. P., Gronbaek, J., \& Danckert, J. (1980). Physical Modelling of Metal Forming Processes. Journal of Applied Metalworking, 1(3), 5-14.

[12] Arthington, M., Cleaver, C., Allwood, J. M., \& Duncan, S. R. (2014). Real-time measurement of ring-rolling geometry using low-cost hardware. Control (CONTROL)

[13] Lin, H., \& Zhi, Z. (1997). The extremum parameters in ring rolling. Journal of Materials Processing Technology, 69, 273-276. 